International Conference on Ceramics, Bikaner, India

International Journal of Modern Physics: Conference Series

Vol. 22 (2013) 724-728

(C) World Scientific Publishing Company

DOI: $10.1142 / \mathrm{S} 2010194513010921$

\title{
STUDY OF ELLIPTIC FLOW AT RHIC ENERGIES
}

\author{
SUDHIR BHARDWAJ \\ Department of Physics, Govt. College of Engineering \& Technology, Bikaner, Rajasthan, INDIA \\ sudhir.hep@gmail.com
}

AJIT K SOMANI

CMRB Govt. Polytechnic College, Sriganganagar, Rajasthan, INDIA ajit.somani@gmail.com

ASHISH AGNIHOTRI

Sri Balaji College of Engineering \& Technology, Jaipur, Rajasthan, INDIA

ashish_agnihotri@yahoo.com

\begin{abstract}
The relativistic heavy ion collisions are the experiment which is working on the different energies 200 and $62.4 \mathrm{GeV}$. In this paper we will study elliptic flow variation with Impact Parameter at above mentioned energies using HIJING event generator models. Elliptic flow is a signal in search of quark gluon plasma.
\end{abstract}

Keywords: Elliptic Flow; Quark Gluon Plasma;

\section{Introduction}

One of the main goals of the heavy ion collision program at the Relativistic Heavy Ion Collider (RHIC) facility is to produce a state of deconfined quarks and gluons, called the Quark Gluon Plasma (QGP), and to study its properties. Elliptic flow $\left(\mathrm{v}_{2}\right)$ measured in heavy-ion collisions are believed to arise because of the pressure gradient developed when two nuclei collides at non-zero impact parameters followed by the subsequent interactions among the constituents (1).

\section{Event Generation}

The simulated data has been generated using HIJING (2) event generator. HIJING is a monte carlo program to study jet and the associated particle production in high energy pp, pA and AA collisions. It is based on QCD-inspired models for jets production with the Lund Model (3) for jet fragmentation. Multiple minijet production, soft excitation, nuclear shadowing of parton distribution functions and jet interaction in dense matter are the physics processes used in HIJING. The event generator is designed mainly to explore the range of possible initial conditions that may occur in relativistic heavy ion collisions. 


\section{Elliptic Flow Determination Technique}

The importance of the value of elliptic flow coefficient, $v_{2}$, to indicate the extent of thermalisation in ultra-relativistic heavy ion collisions, and the large number of experimental measurements led to the need for a variety of methods to determine the value accurately from experimental data $(4 ; 5 ; 6)$. In the more commonly used method the azimuthal distributions are expanded in a Fourier series where the coefficients of expansion are the measures of different orders of anisotropy (4). For small values of these coefficients, the first two terms describe an elliptic shape. The first order anisotropy $\mathrm{v}_{1}$, the directed flow, measures the shift of the centroid of the distribution and is the coefficient of the first term in the expansion. The second order anisotropy $\mathrm{v}_{2}$, the elliptic flow, measures the difference between the major and minor axes of the elliptic shape of the azimuthal distribution and is the coefficient of the second term in the expansion. In the present work, what is now known as the "standard" method of analysis has been used to deter $\psi_{R}$ mine the values of coefficients, and is discussed briefly in the following.

The azimuthal distribution of the emitted particles is characterized by a Fourier expansion about the reaction plane and can be written as "Eq. (1)"

$$
\frac{d N}{d\left(\phi-\psi_{R}\right)} \alpha 1+\sum_{n=1}^{\infty} 2 v_{n} \cos n\left(\phi-\psi_{R}\right)
$$

Here $\varphi$ is the azimuthal angle of the particles and $\psi_{R}$ is known as the reaction plane angle. The reaction plane angle can be known only in simulated data. Experimentally, the reaction plane is estimated by determining the event where the event plane angle is given by "Eq (2)"

$$
\psi_{m}^{\prime}=\frac{1}{m}\left(\tan ^{-1} \frac{\sum w_{i} \sin m \phi_{i}}{\sum w_{i} \cos m \phi_{i}}\right)
$$

Where the summation is over all particles $i$ and the weights $w_{i}$ are all often set to 1 . The azimuthal distribution with respect to the event plane angles can then be expresses as "Eq (3)"

$$
\frac{d N}{d\left(\phi-\psi_{m}^{\prime}\right)} \alpha 1+\sum_{n=1}^{\infty} 2 v_{n m}^{\prime} \cos n m\left(\phi-\psi_{m}^{\prime}\right)
$$

Where the Fourier coefficients of $n^{\text {th }}$ order can be determined from the azimuthal distribution of the particles with respect ti event plane angle of order $\mathrm{m}$, provided $\mathrm{n}$ is an integral multiple of $\mathrm{m}(4)$.

In sub event method we divided the event within two sub event and then estimated the event plane angle from two different event sets so that the inter correlations within the events can be minimized. 


\section{Elliptic Flow Study}

We studied elliptic flow with varying colliding energy. We used Centre of mass energy 62.4 $\mathrm{GeV}$ and $200 \mathrm{GeV}$ for $\mathrm{Cu}+\mathrm{Cu}$ collisions. In Hijing we varied the impact parameter of the collisions from $0 \mathrm{fm}$ to $4 \mathrm{fm}$ with variation of $1 \mathrm{fm}$ for each event set. Number of generated events were 10k. We studied the elliptic flow variation with impact parameter for photon and charge pions. We used two methods for determination of elliptic flow. The first method is event plane method as explained above and the second method is event sub division method in which we divided the event within two sub events and determined the elliptic flow. The results are shown in the following figures.

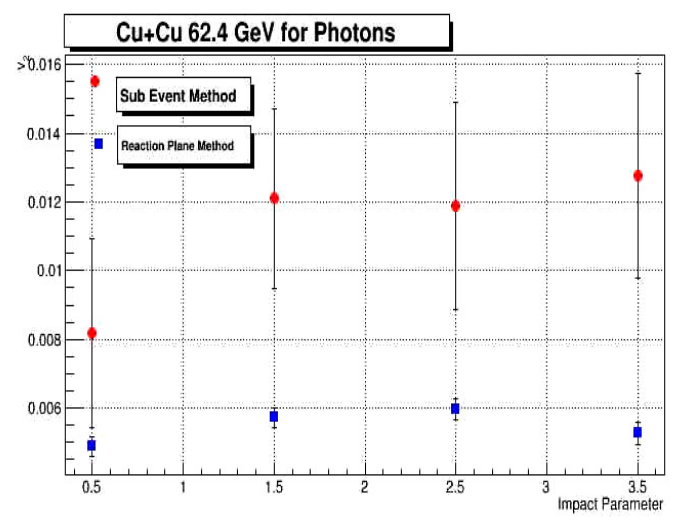

Fig. 1: Elliptic flow variation with Impact Parameter at $\mathrm{Cu}+\mathrm{Cu} 62.4 \mathrm{GeV}$ for Photons

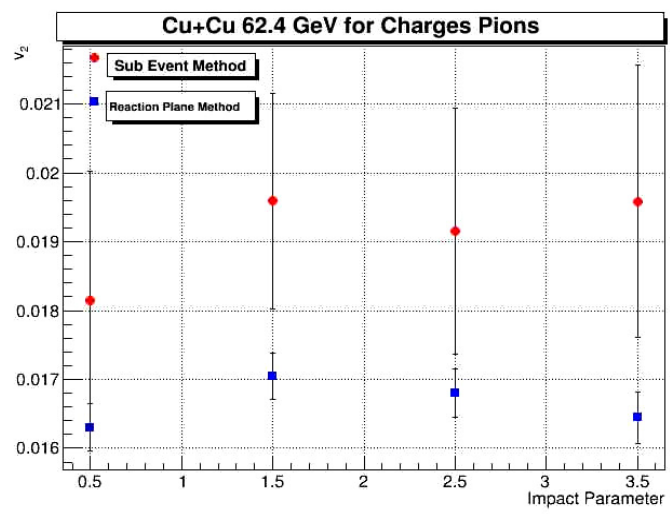

Fig. 2: Elliptic flow variation with Impact Parameter at $\mathrm{Cu}+\mathrm{Cu} 62.4 \mathrm{GeV}$ for Pions 


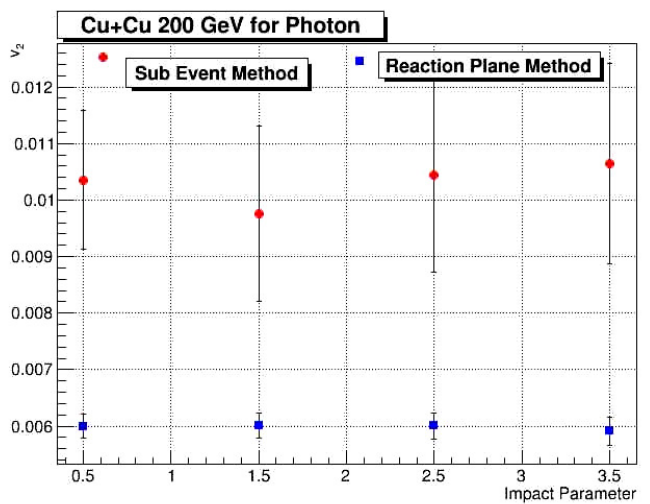

Fig. 3: Elliptic flow variation with Impact Parameter at $\mathrm{Cu}+\mathrm{Cu} 200 \mathrm{GeV}$ for Photons

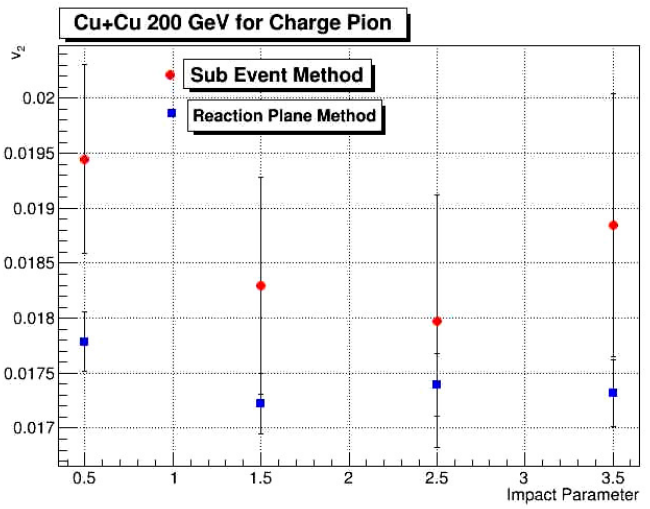

Fig. 4: Elliptic flow variation with Impact Parameter at $\mathrm{Cu}+\mathrm{Cu} 200 \mathrm{GeV}$ for Pions

\section{Conclusions}

By the study of the above figure it is shown clearly that elliptic flow value does not vary much with the changing the impact parameter value. Although the variation of the impact parameter range is very short and the number of events used to estimate elliptic flow is small but these results gives an primary idea of the change of elliptic flow for an random input value of elliptic flow.

We have variation in the elliptic flow value from two different methods. The event plane angle determination from the sub event method is not providing the value of elliptic flow as correct as reaction plane angle provides. 


\section{References}

1. J. Y. Ollitrault, Phys. Rev. D 46, 229 (1992); H. Sorge, Phys. Rev. Lett. 78, 2309 (1997).

2. M. Gyulassy and X. N. Wang, Comput. Phys. Commun. 83 (1994).

3. B. Andersson and G. Gustafson and G. Ingelman and T. Sjostrand, Phys. Rept. 97 (1983).

4. A. M. Poskanzer, S. A. Voloshine, Phys. Rev. C58, 1671-1678 (1998).

5. R. S. Bhalerao et al., Nucl. Phys. A727, 373-426 (2003).

6. N. Borghini and P. M. Dinh and J. Y. Ollitrault, Phys. Rev. C 63, 054906 (2001). 